\title{
Colecistectomía por laparoscopia en colecistitis subaguda: análisis retrospectivo de pacientes en un hospital universitario
}

\author{
Laparoscopic cholecystectomy in subacute cholecystitis: retrospective \\ analysis at a university hospital
}

\author{
Carolina Salinas ${ }^{1}$ Carlos Alberto López¹, Alejandra Ramírez², Ricardo Torres³, \\ María Clara Mendoza ${ }^{4}$, Diana Paola Cuesta ${ }^{5}$
}

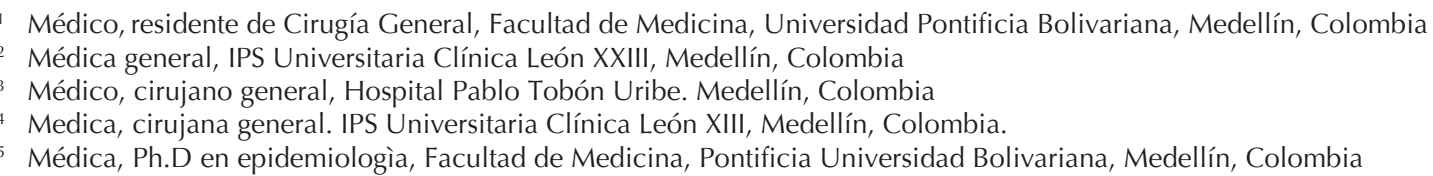

Trabajo presentado en el concurso del residente quirúrgico, 43 Congreso Nacional "Avances en Cirugía", 2017, Medellín, Colombia

\section{Resumen}

Objetivo. Describir los resultados clínicos y quirúrgicos de la colecistectomía por laparoscopia, en pacientes con colecistitis subaguda hospitalizados en una institución universitaria de alta complejidad.

Metodología. Se llevó a cabo un estudio descriptivo y retrospectivo de pacientes adultos con diagnóstico de colecistitis subaguda, intervenidos con colecistectomía por laparoscopia, que fueron hospitalizados en una institución universitaria de alto nivel de complejidad entre enero y diciembre de 20I4. Se evaluaron sus características sociodemográficas, clínicas, del procedimiento, las complicaciones operatorias y a los 30 días, así como la estancia hospitalaria.

Resultados. Se analizaron 184 pacientes con un promedio de edad de 59 años ( $D E=19,34$ ), el 6I \% de sexo femenino. La mayoría de los pacientes (69\%) tenían hallazgos intraoperatorios correspondientes a plastrón, piocolecisto, necrosis y perforación. En I3 \% de los pacientes fue necesaria la conversión a cirugía abierta, y entre las complicaciones se presentó sangrado operatorio en $\mathrm{I3} \%$, fístula biliar en 2,7 \% y lesión de la vía biliar en 0,5\%. La mortalidad intrahospitalaria fue del I,I \%.

Concusiones. La colecistectomía laparoscópica es un procedimiento seguro con una baja tasa de complicaciones, incluso, cuando se practica en casos con más de 72 horas de iniciados los síntomas. Es indispensable que dicho procedimiento sea realizado por un grupo con gran experiencia en cirugía laparoscópica.

Palabras clave: vesícula biliar; colecistitis aguda; colelitiasis; infección; colecistectomía laparoscópica; complicaciones intraoperatorias.

\footnotetext{
Abstract

Objective: To report the clinical results of laparoscopic cholecystectomy in patients with subacute cholecystitis hospitalized at a high complexity level of care university hospital in Medellín, Colombia.

Fecha de recibido: 23/08/2017 - Fecha aceptación: 14/11/2017

Correspondencia: Carolina Salinas, Carrera 27aa \# 36 sur-170, Envigado, Colombia / Teléfono: (312) 297-1688

Correo electrónico: carosp@gmail.com

Citar como: Salinas C, López CA, Ramírez A, Torres R, Mendoza MC, Cuesta DP. Colecistectomía por laparoscopia en colecistitis subaguda: análisis retrospectivo de pacientes en un hospital universitario. Rev Colomb Cir. 2018;33:154-61. https://doi.org/10.30944/20117582.57
} 
Methodology: A retrospective descriptive study was carried out in adult patients diagnosed with subacute cholecystitis who underwent laparoscopic cholecystectomy between January and December 2014 . Sociodemographic, clinical, procedural characteristics, intraoperative complications at 30 days, as well as the hospital stay, were evaluated.

Results: A total of 184 patients met the inclusion criteria, with a mean age of 59 years, predominantly female (6I\%); open surgery conversion rate was $13 \%$, intraoperative bleeding I3\%, biliary fistula 2.7\%, bile duct injury $0.5 \%$, and global mortality I.I\%.

Conclusions: Laparoscopic cholecystectomy is a safe procedure with a low rate of complications, even when performed in patients with more than 72 hours of symptom onset. It is essential that this procedure be performed by a group with high experience in laparoscopic surgery

Keywords: Gallbladder; cholecystitis, acute; cholelithiasis; infection; cholecystectomy, laparoscopic; intraoperative complications.

\section{Introducción}

La colecistitis aguda es una enfermedad inflamatoria de la vesícula, generalmente atribuible a cálculos, sin embargo, se ha asociado a otras causas como isquemia, alteraciones de la motilidad, lesión directa, infección por microorganismos o parásitos, enfermedades del colágeno y reacciones alérgicas. En la mayoría de los pacientes, la causa de esta inflamación es la obstrucción del cuello de la vesícula o del conducto cístico, lo cual genera aumento de la presión intravesicular; según el grado de obstrucción (completa o parcial) y su duración, se determina la gravedad y el pronóstico de la enfermedad. Entre 3,2 \% y I2 \% de los pacientes con colelitiasis desarrolla colecistitis, asociado a un riesgo anual del I a $3 \%$. El $26 \%$ de los pacientes con colelitiasis ingresan al servicio de urgencias por colecistitis aguda ${ }^{2}$.

En el Consenso Internacional de Tokio se clasificó la colecistitis aguda en leve, moderada y grave, de acuerdo con el grado de la inflamación y la presencia de disfunción de órgano, con una proporción de colecistitis aguda grave de 0,6\% y una mortalidad total de o,6 \%. Según esta clasificación, se determina el tratamiento que se debe seguir ${ }^{3}$. Se ha demostrado que el sexo masculino es un factor de riesgo para la inflamación grave de la vesícula biliar y, por tanto, posiblemente es necesaria una intervención más temprana para disminuir el riesgo de complicaciones ${ }^{4}$.

Existe una clasificación de la colecistitis aguda en tres estadios, según el tiempo de evolución después de instaurados los síntomas: aguda, me- nos de 72 horas; subaguda, de 72 horas a I4 días, y crónica, más de I4 días.

Debido al riesgo de complicaciones biliares, como pancreatitis o coledocolitiasis, de progresión a estadios más graves de la enfermedad y de recurrencia de los síntomas, el tratamiento de elección de la colecistitis aguda es la colecistectomía ${ }^{5}$, que esta sea oportuna depende de la gravedad de la colecistitis aguda. Por lo tanto, en la colecistitis aguda leve a moderada, se recomienda la colecistectomía temprana (en las 72 horas de iniciados los síntomas) y, en la colecistitis aguda grave, la elección es la colecistectomía diferida más drenaje vesicular ${ }^{5}$.

La colecistectomía laparoscópica es el abordaje quirúrgico de elección para el manejo de la colecistitis aguda; el $20 \%$ de las colecistectomías laparoscópicas se practican bajo esta indicación ${ }^{6}$. Sin embargo, se debe tener en cuenta que, en presencia de inflamación aguda, el procedimiento quirúrgico es más difícil y, por tanto, mayor la tasa de complicaciones y conversión a cirugía abierta. Esto se debe al edema, las adherencias a otras estructuras u órganos, la distensión vesicular, la friabilidad de los tejidos, la distorsión de la anatomía biliar y vascular hepática, el aumento de la irrigación sanguínea y la congestión ${ }^{7}$. Por esta razón, se han generado algunas controversias con respecto al momento óptimo para practicar la colecistectomía por laparoscopia en pacientes con colecistitis aguda y subaguda.

En casos de colecistitis aguda, múltiples estudios, entre otros estudios aleatorizados y me- 
taanálisis, demuestran que la colecistectomía por laparoscopia temprana es segura, preferiblemente en las primeras 24 horas de iniciados los síntomas. Esto se asocia con menor estancia hospitalaria, menor pérdida sanguínea, menos complicaciones y menos conversiones a cirugía abierta, además de un mayor beneficio económico y una menor dificultad quirúrgica ${ }^{6-14}$, en comparación con la colecistectomía diferida o de intervalo. Esta última conducta consiste en practicar la colecistectomía por laparoscopia entre la sexta y la décimosegunda semana del episodio agudo. Sin embargo, hacerlo así se asocia con fallas en el manejo conservador del $26 \%$ de los pacientes, que obligan a la intervención quirúrgica urgente antes del tiempo previamente estipulado "i; además, el 28,5\% de estos vuelven a ser hospitalizados por complicaciones como colangitis, pancreatitis o perforación vesicular, antes de cumplir el tiempo para la colecistectomía de intervalo 9 .

Existen algunos estudios en los que se comparan los resultados entre pacientes intervenidos en el periodo subagudo de la enfermedad y otros intervenidos en la fase aguda, y se confirma la seguridad de la colecistectomía por laparoscopia después de 72 horas de iniciados los síntomas, sin que se demuestren diferencias estadísticamente significativas en las tasas de conversión, el tiempo quirúrgico, la estancia hospitalaria posoperatoria o la mortalidad 2,15-18.

Debido a la falta de estudios específicos publicados en nuestro medio que incluyan solo casos de colecistitis subaguda (más de 72 horas de iniciado los síntomas), el objetivo de este trabajo fue describir los resultados de la colecistectomía por laparoscopia en este grupo de pacientes, en los cuales se presume que el proceso inflamatorio es mayor.

\section{Materiales y métodos}

Se hizo un estudio observacional y descriptivo, con recolección retrospectiva de datos en la IPS Universitaria-Clínica León XXIII, una institución universitaria de cuarto nivel de complejidad de Medellín, Colombia.
Se incluyeron los pacientes de I8 o más años de edad con colecistopatía calculosa subaguda, sometidos a colecistectomía por laparoscopia entre enero y diciembre del 20I4. Se excluyeron los pacientes con hallazgos operatorios o diagnóstico posterior de neoplasia maligna en la vesícula biliar, con colangitis o con pancreatitis, y aquellos cuya historia clínica no tenía completa la información sobre los resultados de interés. La gravedad de la enfermedad no se clasificó en los pacientes incluidos.

Previa aprobación del Comité de Ética de Investigación en Salud de la Universidad Pontificia Bolivariana y con el aval institucional, se revisó la base de datos de cirugía para identificar los procedimientos de colecistectomía laparoscópica.

Dos investigadores revisaron los criterios de elegibilidad en las historias clínicas y registraron en una base de datos computadorizada las siguientes variables: datos demográficos (edad, sexo, seguridad social y lugar de residencia), comorbilidades (diabetes de tipo 2, enfermedad renal crónica e inmunosupresión), exámenes de laboratorio al momento de la intervención (proteína C reactiva y número de leucocitos), episodios previos de cólico biliar y duración de los síntomas antes del ingreso a la institución; no se logró obtener variables como el peso y la talla, debido a la falta de consignación de estos datos en la historia clínica.

Respecto al procedimiento, se consideraron su duración, los hallazgos operatorios, la necesidad de drenajes, la necesidad de colangiografía y la tasa de conversión a cirugía abierta. Las complicaciones relacionadas con el procedimiento, fueron: fístula biliar, sangrado, infección del sitio operatorio, reintervención o lesión de la vía biliar; además, se consideraron el reingreso antes de los 30 días después del alta hospitalaria, la mortalidad, la estancia hospitalaria y la necesidad de atención en la unidad de cuidados intensivos.

Se describieron las frecuencias de las variables cualitativas. Las variables continuas se expresaron como media con su desviación estándar o como medianas con su rango intercuartílico 
(RIQ), de acuerdo con la distribución de la variable valorada con la prueba de KolmogórovSmirnov. No se hicieron comparaciones para utilizar pruebas estadísticas de hipótesis.

\section{Resultados}

En el periodo de estudio se identificaron 453 colecistectomías laparoscópicas por cualquier causa, y se seleccionaron I84 (4I \%) que cumplían con los criterios de inclusión. En la mayoría de los pacientes no incluidos, su exclusión se debió a que la colecistectomía laparoscópica se practicó en la fase aguda o sin un proceso inflamatorio asociado.

Los pacientes intervenidos tenían una edad promedio de 59 años (desviación estándar, DE=I9,3), y 6I,4 \% fueron mujeres. El 2I \% de los pacientes estaban vinculados al régimen subsidiado del sistema colombiano de seguridad social, y la distribución entre procedencia rural o urbana fue similar.

Las comorbilidades más frecuentes fueron diabetes de tipo 2 (20,I \%), enfermedad renal crónica (IO,3 \%) e inmunosupresión (I,6 \%). La duración de los síntomas antes de la consulta por urgencias fue, en promedio, de cuatro días. En el $34,2 \%$ de los casos existía el antecedente de un cólico biliar antes de la hospitalización.

En los exámenes iniciales de laboratorio tomados en el servicio de urgencias, el promedio de la proteína C reactiva fue de $\mathrm{IO}, \mathrm{I} \mathrm{mg} / \mathrm{dl}$ y, el del número de leucocitos, de $\mathbf{2} .5 \mathrm{I} 6$ por $\mathrm{mm}^{3}$ (tabla I).

Se encontró el antecedente de, por los menos, una cirugía abdominal previa en 57 (3I \%) pacientes, y entre estos la mayoría (9I \%) correspondiente a procedimientos abiertos y localizados en el hemiabdomen inferior.

El tiempo quirúrgico promedio para la colecistectomía laparoscópica fue de 99 minutos ( $\mathrm{DE}=35,4$ minutos). El 89 \% de estos procedimientos fueron practicados en horario diurno.

Se presentó sangrado quirúrgico en 36 (I6\%) pacientes, hubo necesidad de dejar drenajes en 48 (26\%), la tasa de reintervención fue de I,6\%. Se practicaron dos colangiografías operatorias y siete $(3,8 \%)$ colecistectomías subtotales, y en 24
Tabla 1. Características demográficas y clínicas de los pacientes con colecistitis subaguda $(\mathrm{N}=184)$

\begin{tabular}{lc}
\hline \multicolumn{1}{c}{ Característica } & $\mathbf{n}(\%)$ \\
\hline $\begin{array}{l}\text { Demográficas } \\
\text { Edad media en años (desviación } \\
\text { estándar) }\end{array}$ & $59,3(19,3)$ \\
Sexo femenino & $113(61,4)$ \\
Seguridad social contributiva & $145(79,0)$ \\
Lugar de residencia, urbana & $93(50,5)$ \\
$\begin{array}{l}\text { Comorbilidades } \\
\text { Diabetes de tipo 2 }\end{array}$ & $37(20,1)$ \\
Enfermedad renal crónica & $19(10,3)$ \\
$\begin{array}{l}\text { Inmunosupresión } \\
\text { Episodios previos de cólico biliar }\end{array}$ & $3(1,6)$ \\
$\begin{array}{l}\text { Exámenes de laboratorio } \\
\text { Media de la proteína C reactiva } \\
\text { (mg/dl) (desviación estándar) }\end{array}$ & $10(12,58)$ \\
$\begin{array}{l}\text { Recuento de leucocitos por mm3: } \\
\text { media (desviación estándar) }\end{array}$ & $12516(5195)$ \\
$\begin{array}{l}\text { Duración media de síntomas } \\
\text { antes del ingreso (días) } \\
\text { (desviación estándar) }\end{array}$ & $4,2(4,0)$ \\
\hline
\end{tabular}

(I3\%) pacientes hubo necesidad de conversión a colecistectomía abierta. Los hallazgos quirúrgicos más comunes fueron: plastrón vesicular $(24 \%)$, piocolecisto (2I \%) y necrosis vesicular (I6,8 \%); y se encontró perforación vesicular solo en $6,5 \%$ de los pacientes (tabla 2).

La tasa de complicaciones posoperatorias fue de 8,I5 \%, correspondientes a: infección del sitio operatorio, superficial en dos (I,I \%) y de órgano o espacio en dos (I,I\%); fístula biliar en cinco $(2,7 \%)$ y lesión de la vía biliar en uno (0,5\%) (tabla 3).

Del total de I84 pacientes, 23 (I2 \%) requirieron cuidados en las unidades de cuidados intermedios o intensivos: un paciente $(0,54 \%)$ por hemoperitoneo posoperatorio, cuatro $(2, \mathrm{I} \%)$ por choque séptico y los otros I8 (9,7\%) por descompensación de sus comorbilidades de base o por procesos agudos diferentes a la colecistitis.

Se presentó una tasa de mortalidad de I,I \%, correspondiente a dos pacientes fallecidos, uno 
Tabla 2. Características de la colecistectomía por laparoscopia en pacientes con colecistitis subaguda $(\mathrm{N}=184)$

\begin{tabular}{lc}
\hline Características del procedimiento & $\mathbf{n}(\%)$ \\
\hline $\begin{array}{l}\text { Media de tiempo quirúrgico (minutos) } \\
\text { (desviación estándar) }\end{array}$ & $99(35,4)$ \\
Colangiografía intraoperatoria & $2(1,1)$ \\
$\begin{array}{l}\text { Traslado unidad cuidados especiales/ } \\
\text { intensivos }\end{array}$ & $22(12,0)$ \\
Hallazgos intraoperatorios & \\
Plastrón & $45(24,5)$ \\
Piocolecisto & $39(21,2)$ \\
Necrosis & $31(16,8)$ \\
Perforación & $12(6,5)$ \\
\hline
\end{tabular}

Tabla 3. Complicaciones de la colecistectomía por laparoscopia en pacientes con colecistitis subaguda $(\mathrm{N}=184)$

\begin{tabular}{ll}
\hline Complicaciones & n (\%) \\
\hline Sangrado & $30(16,3)$ \\
Conversión a cirugía abierta & $24(13,0)$ \\
Colecistectomía subtotal & $7(3,8)$ \\
Reingreso a 30 días & $7(3,8)$ \\
Fístula biliar & $5(2,7)$ \\
Infección del sitio operatorio & $4(2,2)$ \\
$\quad$ Superficial & $2(1,1)$ \\
Profunda & $0(0,0)$ \\
$\quad$ Órgano/espacio & $2(1,1)$ \\
Reintervención a 30 días & $3(1,6)$ \\
Muerte intrahospitalaria & $2(1,1)$ \\
Lesión de la vía biliar & $1(0,5)$ \\
\hline
\end{tabular}

por paro cardiorrespiratorio durante la cirugía y el otro por sepsis biliar resistente (tabla 3).

La tasa de reingreso fue del 3,8\%, siendo el dolor abdominal su principal causa; la tasa de reintervención a 30 días fue de 1,6 \% (3 casos). La mediana de la estancia hospitalaria total fue de 6 días, con un rango que varió de 4 a io días, mientras que la mediana de la estancia hospitalaria posquirúrgica fue de un día, con un rango que varió de I a 3 días (tabla 4).
Tabla 4. Estancia hospitalaria en pacientes con colecistitis subaguda $(\mathrm{N}=184)$

\begin{tabular}{lc}
\hline Estancia hospitalaria (días) & Mediana (RIQ) \\
\hline Prequirúrgica & $4(3-6)$ \\
Posquirúrgica & $1(1-3)$ \\
Total & $6(4-10)$ \\
\hline
\end{tabular}

RIQ: rango intercuartil

\section{Discusión}

La colecistitis aguda continúa siendo una importante causa de consulta en los servicios de urgencias, lo que mantiene a la colecistectomía por laparoscopia como uno de los procedimientos más comúnmente practicados por los cirujanos generales ${ }^{1}$. En las últimas décadas, ha persistido el debate sobre el momento adecuado para practicar este procedimiento ${ }^{2}$, especialmente, porque se considera que, a mayor tiempo de evolución del proceso inflamatorio, existe un mayor riesgo de complicaciones derivadas de la intervención por la formación de adherencias, abscesos y fibrosis perivesicular. Zhu, et al., propusieron el concepto de " 72 horas de oro" para la práctica de la colecistectomía por laparoscopia, basados en estudios retrospectivos ${ }^{4}$.

Con el avance y la difusión de técnicas mínimamente invasivas, la colecistectomía por laparoscopia se ha convertido en un procedimiento ampliamente practicado por cirujanos tratantes y en entrenamiento ${ }^{6,7}$, lo que puede llegar a contribuir a una depuración de la técnica que conlleve menor tasa de complicaciones en todos los pacientes en quienes se utilice.

Múltiples estudios, incluidos estudios aleatorizados y metaanálisis, han demostrado que la colecistectomía temprana por laparoscopia es segura en casos de colecistitis aguda, en especial, si se practica en las primeras 24 horas de iniciados los síntomas, asociándose a menor estancia hospitalaria, menor pérdida sanguínea, menos complicaciones y conversión a cirugía abierta, más beneficio económico y menor dificultad quirúrgica ${ }^{6-14}$. No obstante, al comparar los resultados de la intervención en fase subaguda y 
en fase aguda, se confirma la seguridad de esta técnica quirúrgica después de 72 horas de instaurados los síntomas y no se observan diferencias estadísticamente significativas en las tasas de conversión, el tiempo quirúrgico, la estancia hospitalaria posoperatoria o la mortalidad ${ }^{2,15-18}$.

En estudios más recientes en centros con experiencia en cirugía mínimamente invasiva, se ha demostrado que el practicar la cirugía temprana (en los 7 primeros días de iniciados los síntomas), es mejor que hacerlo en forma diferida (entre 6 y 22 semanas después), en términos de costoefectividad y calidad de vida, sin que se aumente la tasa de complicaciones ${ }^{19}$. En el estudio de Tan, et al., se encontró que solo el 43,I \% de los pacientes eran intervenidos en las primeras 72 horas de iniciado los síntomas; además, se demostró un aumento significativo en la tasa de conversión a cirugía abierta cuando la colecistectomía laparoscópica se practicaba después de 7 días (2I Vs. 8 \%). Postularon que la alta tasa de conversión hallada por otros autores en pacientes operados después de las primeras 72 horas, correspondía a un subgrupo con síntomas de más de una semana, debido a la dificultad en precisar el inicio de los síntomas en algunos de ellos, lo que ampliaba aún más el tiempo para practicar la colecistectomía por laparoscopia durante la fase aguda y la subaguda. En la Universidad de Toho en Tokyo, los resultados fueron similares en un análisis retrospectivo de 224 pacientes; en la colecistectomía por laparoscopia practicada después de 7 días de iniciados los síntomas, el tiempo quirúrgico y la estancia hospitalaria fueron más prolongados: 95 (30-295) Vs. II4 (20-242) minutos, y 5 (3-4I) Vs. 9 (3-32) días, respectivamente ${ }^{20}$.

En el presente estudio, se evaluó la seguridad de practicar la colecistectomía por laparoscopia en un grupo de pacientes con más de 72 horas de iniciados los síntomas. Se evaluaron 184 pacientes, $69 \%$ de ellos con hallazgos intraoperatorios indicativos de un importante proceso inflamatorio (plastrón, piocolecisto, necrosis o perforación), presumiendo una mayor dificultad técnica para el cirujano. Sin embargo, la media de tiempo quirúrgico fue de 99 minutos, siempre contando con la participación de un residente de cirugía general; este tiempo es un poco mayor al reportado en la revisión Cochrane ${ }^{17}$. Es de resaltar que, en los pacientes operados en los primeros 7 días, el tiempo quirúrgico fue mayor, tal como se encontró en estudios similares ya citados ${ }^{19-20}$.

En 7 de los I84 pacientes, se practicó colecistectomía subtotal, similar a lo reportado en otros estudios ${ }^{(21)}$; por lo tanto, se puede considerar una técnica segura en manos de cirujanos experimentados en técnicas mínimamente invasivas.

Se presentó algún tipo de complicación en $4 \mathrm{I}, 2 \%$ de los pacientes y la más frecuente fue el sangrado, en 30, la mayoría de los cuales requirió drenaje abdominal, mientras que solo un paciente debió reintervenirse.

La tasa global de conversión a cirugía abierta fue de $13 \%$, mientras que esta tasa varía entre 6,2 y $15 \%$ en otras series; se asocia directamente con la experiencia del cirujano en la solución de problemas por dicha vía de abordaje. La lesión de la vía biliar es la complicación más temida de la colecistectomía por laparoscopia, por su importante impacto en la calidad de vida y la gran morbilidad asociada; sólo un paciente la presentó en el presente estudio. Se encontró una tasa de mortalidad de I,I \% (dos pacientes), uno por paro intraoperatorio y el otro por choque séptico y falla multiorgánica.

Entre otros resultados, como estancia hospitalaria, complicaciones asociadas, reingreso e infección del sitio operatorio, no hubo diferencias significativas al compararlos con estudios similares reportados en la literatura científica.

Este estudio estuvo limitado por el pequeño número de pacientes estudiados y su diseño observacional retrospectivo y, además, por la heterogeneidad del grupo quirúrgico involucrado (más de I2 cirujanos con diferente experiencia quirúrgica), lo que conlleva la heterogeneidad de los resultados, sin embargo, cabe resaltar que existe evidencia en la literatura de que un adecuado entrenamiento en colecistectomía por laparoscopia en cirujanos generales, tiene tasas estables y comparables con otros estudios de conversion abierta, estancia hospitalaria y complicaciones ${ }^{22}$. 
El dolor abdominal agudo causa 5-I0\% de los ingresos por urgencias y su localizacion en hipocondrio derecho aquivale a la tercera causa de consulta en las estadísticas nacionales, con una alta prevalencia de enfermedades de la vesícula biliar en el servicio de urgencias ${ }^{23}$. Estos estudios son importantes porque orientan las decisiones del manejo médico-quirúrgico de nuestros pacientes

En conclusión, la colecistectomía laparoscópica es un procedimiento seguro con una baja tasa de complicaciones, incluso, cuando se practica en pacientes con más de 72 horas de sintomatología; por esta razón, apoyado en revisiones científicas ${ }^{24}$, se considera que el manejo médico de estos pacientes con más de 72 horas de síntomas, conlleva un mayor costo para el sistema de salud, estancia hospitalaria más prolongada, mayor duración de los antibióticos, mayor morbilidad y, por tanto, tasa más alta de reingresos. Es indispensable que la colecistectomía por laparoscopia sea practicada por un grupo con experiencia en cirugía laparoscópica, lo que claramente ha demostrado una menor tasa de complicaciones. Vale la pena resaltar la baja tasa de lesiones de la vía biliar encontrada en el grupo de estudio, lo que apoya su manejo quirúrgico. Se requieren estudios con mayor poder estadístico que nos permitan recomendar la colecistectomía por laparoscopia en una etapa subaguda de la enfermedad

\section{Agradecimientos}

Al personal de salud y de docencia e investigación de la IPS Universitaria-Clínica León XXIII de Medellín, Colombia, por su apoyo en la ejecución de esta investigación.

\section{Declaración de conflicto de intereses}

Los autores declaran no tener ningún conflicto de intereses.

\section{Referencias}

I. Kimura Y, Takada T, Strasberg SM, Pitt HA, Gouma DJ, Garden OJ, et al. TGi3 current terminology, etiology, and epidemiology of acute cholangitis and cholecystitis. J Hepatobiliary Pancreat Sci. 2013;20:8-23.
2. Farooq T, Buchanan G, Manda V, Kennedy R, Ockrim J. Is early laparoscopic cholecystectomy safe after the "safe period"? J Laparoendosc Adv Surg Tech A. 2009;19:47I-4.

3. Yokoe M, Takada T, Strasberg SM, Solomkin JS, Mayumi T, Gomi H, et al. TGI3 diagnostic criteria and severity grading of acute cholecystitis (with videos). J Hepatobiliary Pancreat Sci. 2013;20:35-46.

4. Zhu B, Zhang Z, Wang Y, Gong K, Lu Y, Zhang N. Comparison of laparoscopic cholecystectomy for acute cholecystitis within and beyond $72 \mathrm{~h}$ of symptom onset during emergency admissions. World J Surg. 20I2;36:2654-8.

5. Yamashita Y, Takada T, Strasberg SM, Pitt HA, Gouma DJ, Garden OJ, et al. TGi3 surgical management of acute cholecystitis. J Hepatobiliary Pancreat Sci. 2013;20:89-96.

6. Casillas RA, Yegiyants S, Collins JC. Early laparoscopic cholecystectomy is the preferred management of acute cholecystitis. Arch Surg. 2008;143:533-7.

7. Agrawal R, Sood KC, Agarwal B. Evaluation of early versus delayed laparoscopic cholecystectomy in acute cholecystitis. Surg Res Pr. 2015;2015:I-7. doi: IO.II55/20I5/34980I.

8. Chandler CF, Lane JS, Ferguson P, Thompson JE, Ashley SW. Prospective evaluation of early versus delayed laparoscopic cholecystectomy for treatment of acute cholecystitis. Am Surg. 2000;66:896-900.

9. Cheruvu CVN, Eyre-Brook IA. Consequences of prolonged wait before gallbladder surgery. Ann R Coll Surg Engl. 2002;84:20-2.

Io. Gutt CN, Encke J, Köninger J, Harnoss J-C, Weigand K, Kipfmüller K, et al. Acute cholecystitis. Ann Surg. 2013;258:385-93.

II. Johansson M, Thune A, Blomqvist|A, Nelvin L, Lundell L. Management of acute cholecystitis in the laparoscopic era: Results of a prospective, randomized clinical trial. J Gastrointest Surg. 2003;7:642-5.

I2. Hershkovitz Y, Kais H, Halevy A, Lavy R. Interval laparoscopic cholecystectomy: What is the best timing for surgery? Isr Med Assoc J. 20I6;I8:I0-2.

I3. Lai P, Kwong K, Leung K, Kwok S, Chan A. Randomized trial of early versus delayed laparoscopic cholecystectomy for acute cholecystitis. Br J Surg. 1998;85:764-7.

I4. Lo CM, Liu CL, Fan ST, Lai EC, Wong J. Prospective randomized study of early versus delayed laparoscopic cholecystectomy for acute cholecystitis. Ann Surg. I998;227:46I-7.

15. Ohta M, Iwashita Y, Yada K, Ogawa T, Kai S, Ishio T, et al. Operative timing of laparoscopic cholecystectomy for acute cholecystitis in a Japanese Institute. J Soc Laparoendosc Surg. 2012;16:65-70.

I6. Gomes RM, Mehta NT, Varik V, Doctor NH. No 72-hour pathological boundary for safe early laparoscopic cho- 
lecystectomy in acute cholecystitis: A clinicopathological study. Ann Gastroenterol. 2013;26:340-5.

I7. Gurusamy KS, Davidson C, Gluud C, Davidson BR. Early versus delayed laparoscopic cholecystectomy for people with acute cholecystitis. Cochrane Database Syst Rev. 2013;6(6).

I8. Ambe PC, Weber S a, Wassenberg D. Is gallbladder inflammation more severe in male patients presenting with acute cholecystitis? BMC Surg. 2015;15:4-7.

I9. Tan JK, Goh JC, Lim JW, Shridhar IG, Madhavan K, Kow AW. Same admission laparoscopic cholecystectomy for acute cholecystitis: is the "golden 72 hours" rule still relevant? HPB (Oxford). 20I7;19:47-5I.

20. Asai K, Watanabe M, Kusachi S, Matsukiyo H, Saito $\mathrm{T}$, Ishii T, et al.. Evaluating the timing of laparoscopic cholecystectomy for acute cholecystitis in an experienced center based on propensity score matching. Asian J Endosc Surg. 20I7;10:I66-72.
2I Kuwabara J, Watanabe Y, Kameoka K, Horiuchi A, Sato $\mathrm{K}$, Yukumi S, et al. Usefulness of laparoscopic subtotal cholecystectomy with operative cholangiography for severe cholecystitis. Surg Today. 20I4;44:462-5.

22. Díaz S, Correa MJ, Giraldo LM, Ríos DC, Solórzano F, Wolff JD, et al. Experiencia en colecistectomía por laparoscopia en la Clínica Universitaria CES. Rev Colomb Cir. 20I2;27:275-80.

23. Bejarano M, Gallego CX, Gomez JR. Frecuencia de abdomen agudo quirúrgico en pacientes que consultan al servicio de urgencias. Rev Colomb Cir. 20II;26:33-4I.

24. Roulin D, Saadi A, Di Mare L, Demartines N, Halkic N. Early versus delayed cholecystectomy for acute cholecystitis, are the 72 hours still the rule?: A randomized trial. Ann Surg. 2016;264:717-22. 